Check for updates

Cite this: Mater. Adv., 2022, 3, 3192

Received 25th November 2021 Accepted 22nd February 2022

DOI: $10.1039 / \mathrm{d} 1 \mathrm{ma01113h}$

rsc.li/materials-advances

\title{
Do electron distributions with orbital degree of freedom exhibit anisotropy?†
}

\author{
Taishun Manjo, (D) a Shunsuke Kitou, ${ }^{\mathrm{b}}$ Naoyuki Katayama, ${ }^{a}$ Shin Nakamura, ${ }^{\mathrm{c}}$ \\ Takuro Katsufuji, ${ }^{d}$ Yoichi Nii, ${ }^{\text {ef }}$ Taka-hisa Arima, (D) bo Joji Nasu, ${ }^{\text {fh }}$ Takumi Hasegawa, \\ Kunihisa Sugimoto, (D) Daisuke Ishikawa, ${ }^{\mathrm{kl}}$ Alfred Q. R. Baron ${ }^{\mathrm{kl}}$ and Hiroshi Sawa*a
}

\begin{abstract}
Orbital degrees of freedom appear in partially filled degenerate orbitals and are responsible for a variety of physical properties. Although many methods for directly observing orbital states in ordered states have been proposed, it is difficult to determine degenerate orbital states directly. We found that highprecision diffraction data obtained by synchrotron radiation $\mathrm{X}$-rays can be used to observe only the valence electron density distribution with minimal analytical bias. The results obtained using this method clearly showed the anisotropy of the partially filled degenerate $3 \mathrm{~d}$ orbital states in spinel vanadium oxide $\mathrm{FeV}_{2} \mathrm{O}_{4}$. The $3 \mathrm{~d}$ orbital quantum state was successfully determined from this anisotropy.
\end{abstract}

\section{Introduction}

The interplay of charge, spin, orbital, and lattice degrees of freedom driven by electron correlation is a unifying principle across a wide range of transition metal systems. ${ }^{1-5}$ Such a picture provides a framework for determining whether oneelectron physics is inadequate in a particular situation. The orbital degree of freedom in partially filled d-electron orbitals and related aspects such as orbital order, orbital waves, specific magnetic properties, and insulator-metal transitions has aroused considerable interest. Therefore, several methods have been proposed to observe orbital states, such as high harmonics generated from femtosecond laser pulses, ${ }^{6}$ ultraviolet angle-resolved photoelectron spectroscopy, ${ }^{7}$ resonant X-ray scattering, ${ }^{8}$ and non-resonant inelastic synchrotron X-ray

\footnotetext{
${ }^{a}$ Department of Applied Physics, Nagoya University, Nagoya 464-8603, Japan.

E-mail: z47827a@cc.nagoya-u.ac.jp

${ }^{b}$ RIKEN Center for Emergent Matter Science, Wako 351-0198, Japan

${ }^{c}$ Department of Science and Engineering, Teikyo University, Utsunomiya 320-8551,

Japan

${ }^{d}$ Department of Physics, Waseda University, Shinjuku 169-8555, Japan

${ }^{e}$ Institute for Materials Research, Tohoku University, Sendai 980-8577, Japan

${ }^{f}$ PRESTO, Japan Science and Technology Agency (JST), Kawaguchi 332-0012, Japan

${ }^{g}$ Department of Advanced Materials Science, The University of Tokyo, Kashiwa 2778561, Japan

${ }^{h}$ Department of Physics, Tohoku University, Sendai 980-8578, Japan

${ }^{i}$ Graduate School of Advanced Science and Engineering, Hiroshima University, Higashi-Hiroshima 739-8521, Japan

${ }^{j}$ Japan Synchrotron Radiation Research Institute (JASRI), SPring-8, Hyogo 679-5198, Japan

${ }^{k}$ Precision Spectroscopy Division, CSRR, SPring-8/JASRI, Hyogo 679-5198, Japan

${ }^{l}$ Materials Dynamics Laboratory, RIKEN SPring-8 Center, Hyogo 679-5198, Japan

$\dagger$ Electronic supplementary information (ESI) available. See DOI: 10.1039/ d1ma01113h
}

scattering. ${ }^{9}$ Despite the need to observe the orbital degrees of freedom, these experimental methods only allow for determining the orbital ordered or stable state. Even first-principles calculations cannot easily determine metastable states at finite temperatures. Orbital degrees of freedom are realized when degenerate orbitals are partially filled, but since there are few reports of their observation, the electron distribution in degenerate orbitals is only vaguely understood. In the high-temperature region, the anisotropy of degenerate orbitals is expected to be suppressed due to thermal fluctuations caused by the surrounding atoms and crystal field. The anisotropy of two or more degenerate orbitals would still not be uniquely determined, since orbitals with different symmetries are hybridized by quantum mechanical interference. Therefore, it has been widely believed that the degeneracy of localized orbitals blurs anisotropic electronic distributions. However, this physical prediction is yet to be confirmed.

We aimed to observe the valence electron density (VED) distribution to determine the orbital state. Our proposed core differential Fourier synthesis (CDFS) method ${ }^{10,11}$ allows us to directly observe the 3D distribution of the VED by synchrotron X-ray diffraction (XRD). This method calculates the VED; $\rho_{\nu}(r)$ in the unit cell by subtracting the structure factor calculated using only the core-shell electrons from the experimental structure factor by the XRD, and then inverse Fourier transforming this difference structure factor as shown in eqn (1). The CDFS is an innovative method that exploits a blind spot in the analysis of diffraction data by minimizing the effect of censoring of the inverse Fourier transform with finite coefficients, which is usually a problem (Fig. S1, ESI $\dagger$ ). In eqn (1), $\boldsymbol{K}$ is the scattering vector. $F_{\text {obs }}|(\boldsymbol{K})|$ is the absolute value of the experimentally observed crystal structure factor. $f_{j}^{\text {core }}$ is the $j$ th atomic scattering factor with only the core electrons 
contribution. There are several publicly available databases of atomic scattering factors $f^{\text {core }}$ for these core-shell electrons. $T_{j}$ is the $j$ th atomic displacement parameter. $P$ and $P^{\text {core }}$ represent the phase factor. $P^{\text {core }}$ is with only the core electrons contribution calculated as $P^{\text {core }}=F_{\text {cal }}^{\text {core }}(K) /\left|F_{\text {cal }}^{\text {core }}(K)\right| . n_{v}$ is the total number of valence electrons contained in the unit cell. Since $|F(000)|$ is not experimentally observable, we add the $n_{\nu} / V$ term to correct the VED distribution data.

$$
\begin{aligned}
& \rho_{v}(\boldsymbol{r}) \\
& \quad=\frac{1}{V} \sum_{\boldsymbol{K}}\left[\left(\left|F_{\mathrm{obs}}(\boldsymbol{K})\right| P-\left|\sum_{j} f_{j}^{\mathrm{core}}(\boldsymbol{K}) T_{j} e^{-i \boldsymbol{K} \cdot \boldsymbol{r}_{j}}\right| P^{\mathrm{core}}\right) e^{i \boldsymbol{K} \cdot \boldsymbol{r}_{j}}\right]+\frac{n_{v}}{V}
\end{aligned}
$$

Based on our experience of the endohedral proof of $\mathrm{H}_{2}$ @ATOCF (open-cage $\mathrm{C}_{60}$ ) ${ }^{12}$ and $\mathrm{Li}^{+} @ \mathrm{C}_{60}{ }^{13}$ the thin VED analysis requires high-brightness synchrotron XRD data. However, the most noteworthy aspect of this method is that the VED analysis can be performed simply by using diffraction data measured with appropriate short-wavelength X-rays, and no assumptions, such as a model of valence electrons, are required.

The configuration of the partially occupied d orbitals leads to peculiar magnetic and electronic transitions, such as the Verwey transition of $\mathrm{Fe}_{3} \mathrm{O}_{4} \cdot{ }^{14}$ In this study, we focus on the orbital state of vanadium spinel oxide $\mathrm{FeV}_{2} \mathrm{O}_{4}$, which has an isomorphous structure as magnetite, in the high-temperature phase. In this system, despite the lack of charge degrees of freedom due to the Mott insulator, both electronic correlations and geometric frustration are present, resulting in an interplay between the different degrees of freedom, such as spin, orbital, and lattice, which leads to three successive phase transitions with decreasing temperature. ${ }^{15-24} \mathrm{FeV}_{2} \mathrm{O}_{4}$ has been widely studied from both experimental and theoretical perspectives; however, owing to the intertwining of the multiple degrees of freedom, it is difficult to understand the orbital state completely. $\mathrm{AV}_{2} \mathrm{O}_{4}$ with other cations at the Fe site has been studied to avoid multiple degrees of freedom. For example, nonmagnetic A-site $\left(\mathrm{A}=\mathrm{Mg},{ }^{25} \mathrm{Zn},{ }^{26} \mathrm{Cd}^{27}\right)$ and magnetic but orbitally inactive A-site $\left(\mathrm{A}=\mathrm{Mn},{ }^{28} \mathrm{Co}^{29}\right)$ have been studied. The role of Fe was also studied in the isomorphic material $\mathrm{FeCr}_{2} \mathrm{O}_{4} \cdot{ }^{30}$ By integrating these studies, the crystal structure of $\mathrm{FeV}_{2} \mathrm{O}_{4}$ is predicted to change from cubic to tetragonal $(a>c)$ at $140 \mathrm{~K}$ due to $\mathrm{Fe}$ orbital ordering, to orthorhombic at $110 \mathrm{~K}$ due to Fe-spinorbital interaction, and to tetragonal $(a<c)$ at $65 \mathrm{~K}$ due to $\mathrm{V}$ orbital ordering. Although there has been considerable discussion on the origin of the phase transition, mainly focusing on the orbital ordered state of $\mathrm{V}$, there are few reports that have definitively determined the electronic state of the highsymmetry cubic phase.

In $\mathrm{FeV}_{2} \mathrm{O}_{4}$, because the formal charges of $\mathrm{Fe}$ and $\mathrm{V}$ are +2 and +3 , the electron configurations are $3 \mathrm{~d}^{6}$ and $3 \mathrm{~d}^{2}$, respectively. The $S=2$ spin at the Fe site and the $S=1$ spin at the V site is determined by the magnetization experiment. ${ }^{16}$ The unit cell consists of a 3D structure of $8 \mathrm{FeO}_{4}$ tetrahedra and $16 \mathrm{VO}_{6}$ octahedra (Fig. 1A). This system has three structural phase transitions with decreasing temperature, but there is only one
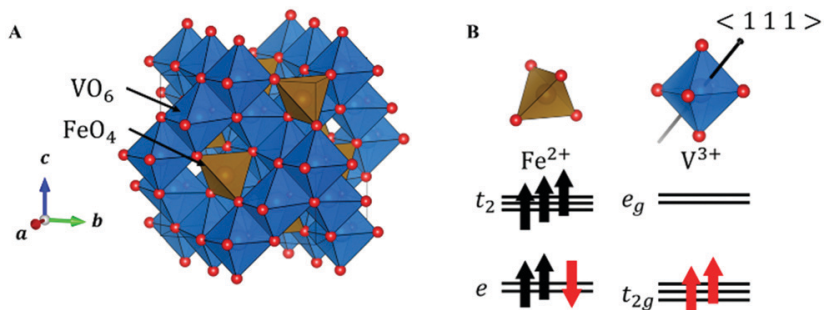

Fig. 1 (A) Crystal structure of $\mathrm{FeV}_{2} \mathrm{O}_{4}$ in the cubic phase. (B) The orbital states of $\mathrm{V}$ and $\mathrm{Fe}$ in the cubic phase. The black arrow in $\mathrm{VO}_{6}$ indicates the $\left\langle\begin{array}{llll}1 & 1 & 1\rangle \text { direction. }\end{array}\right.$

crystallographically independent site each for $\mathrm{Fe}, \mathrm{V}$, and $\mathrm{O}$ in all phases. In the cubic phase of the space group $F d \overline{3} m$, because $\mathrm{FeO}_{4}$ is a regular tetrahedron, the $3 \mathrm{~d}$ orbitals of $\mathrm{Fe}^{2+}$ split into lower doubly degenerate e orbitals and higher triply degenerate $t_{2}$ orbitals. Owing to the high-spin configuration, five electrons with an up-spin form the half-closed shell configuration, and one "down-spin electron" on the e orbital is responsible for the orbital degree of freedom (Fig. 1B). Therefore, $\mathrm{Fe}^{2+}$ has both spin and orbital degrees of freedom. In contrast, the octahedral site of $\mathrm{VO}_{6}$ has inversion symmetry, and the $3 \mathrm{~d}$ orbitals of $\mathrm{V}^{3+}$ are divided into lower triply degenerate $t_{2 g}$ orbitals and higher doubly degenerate $\mathrm{e}_{\mathrm{g}}$ orbitals. If $\mathrm{VO}_{6}$ forms a regular octahedron $\left(\mathrm{O}_{\mathrm{h}}\right)$, then $3 \mathrm{~d}^{2}$ electrons occupy triple degenerate $t_{2 \mathrm{~g}}$ orbitals in a high-spin configuration (Fig. 1B), and $\mathrm{V}^{3+}$ has spin and orbital degrees of freedom. However, the $3 \mathrm{~d}$ state is complicated because of a slight $D_{3 \mathrm{~d}}$ distortion in the $\mathrm{VO}_{6}$ octahedron. Therefore, the $\mathrm{FeV}_{2} \mathrm{O}_{4}$ system has spin and orbital degrees of freedom at both the $\mathrm{Fe}^{2+}$ and $\mathrm{V}^{3+}$ sites.

The $\mathrm{VO}_{6}$ octahedron has a $D_{3 \mathrm{~d}}$-type distortion that satisfies the.$\overline{3} m$ site symmetry at the $\mathrm{V}$ site. The triply degenerate $t_{2 \mathrm{~g}}$ orbitals are further split into one orbital and two degenerate orbitals by the crystal field formed by the surrounding anions. One orbital wave function is uniquely described by the following formula because the $\left\langle\begin{array}{llll}1 & 1 & 1\end{array}\right\rangle$ direction is the quantum axis.

$$
\varphi_{1}(\boldsymbol{r})=\frac{1}{\sqrt{3}}(|y z\rangle+|z x\rangle+|x y\rangle)
$$

In this formula, $\boldsymbol{x}, \boldsymbol{y}$, and $\boldsymbol{z}$ are determined to be parallel to lattice vectors $\boldsymbol{a}, \boldsymbol{b}$, and $\boldsymbol{c}$, respectively. In contrast, the two wavefunctions of doubly degenerate orbitals of $t_{2 g}$ are not uniquely described. When these two orbital wavefunctions are denoted by $\varphi_{2}$ and $\varphi_{3}$, three wave functions, $\varphi_{1}, \varphi_{2}$ and $\varphi_{3}$, in $t_{2 g}$ have to be orthogonal to each other. Determining the energy level of the post-split orbital is difficult because of the small $D_{3 \mathrm{~d}}$-type distortion. There are no charge degrees of freedom and the volume of the $\mathrm{VO}_{6}$ octahedron is conserved. In the case of $\mathrm{FeV}_{2} \mathrm{O}_{4}$ in the cubic phase, the octahedron of $\mathrm{VO}_{6}$ extends in the $\left\langle\begin{array}{lll}1 & 1 & 1\end{array}\right\rangle$ direction, and the area of the oxygen triangular surface perpendicular to the quantum axis is smaller than that of the regular octahedron. When calculating the $\mathrm{V}^{3+}-3 \mathrm{~d}^{2}$ orbital state using the $Q$-mode analysis, ${ }^{31}$ the $\mathrm{t}_{2 \mathrm{~g}}$ orbitals split into lower doubly degenerate orbitals and higher $\varphi_{1}$ orbital. In contrast, when calculating the $\mathrm{V}^{3+}-3 \mathrm{~d}^{2}$ orbital state using a 
point-charge model with the Ewald method ${ }^{32}$ (for details of the calculation method, see ref. (10)), the $t_{2 g}$ orbitals split into the lower $\varphi_{1}$ orbital and higher doubly degenerate orbitals. It is important to note that the triple degeneracy of the $t_{2 g}$ orbitals is maintained when the effect of the $D_{3 \mathrm{~d}}$-type strain is considerably weaker than that of the thermal fluctuation. Therefore, we attempted to determine the correct orbital state from the 3D distribution of VED obtained by the CDFS method.

\section{Crystal structure and results of CDFS method}

XRD experiments were performed using single crystals on the BL02B1 beamline at the synchrotron facility SPring-8 in Japan [see ESI $\dagger$ Section 1]. Fig. $2 \mathrm{D}$ and $4 \mathrm{~B}$ show the observed VED around the $\mathrm{V}(1 / 21 / 21 / 2)$ and the $\mathrm{Fe}(1 / 8,1 / 8,1 / 8)$ sites at $160 \mathrm{~K}$. The VED around the $\mathrm{V}$ site is more clearly anisotropic than the Fe site. The reason is that only $1 / 6$ of the $3 \mathrm{~d}$ electrons contribute to the anisotropy in the $\mathrm{Fe}$ site, whereas the $\mathrm{V}$ site directly reflects the anisotropy of the $3 \mathrm{~d}^{2}$ electrons. The anisotropy of the VED at the V site was considered as follows:

We defined $\varphi_{2}$ and $\varphi_{3}$ such that that the three wavefunctions, including $\varphi_{1}$, are orthogonal to simulate the $3 \mathrm{D}$ VED distribution.

$$
\left\{\begin{array}{l}
\varphi_{2}(\boldsymbol{r})=\frac{1}{\sqrt{2}}(|y z\rangle-|z x\rangle) \\
\varphi_{3}(\boldsymbol{r})=\frac{1}{\sqrt{6}}(|y z\rangle+|z x\rangle-2|x y\rangle)
\end{array}\right.
$$

When a valence electron occupies the $\varphi_{i}$ orbital, $\left|\varphi_{i}\right|^{2}$ corresponds to VED. Because the CDFS method extracts only the VED, information on the phase of the orbital wave function is not obtained. Here, we expected the effect of the orbital angular moment $l$ to be negligible and focused mainly on the Jahn-Teller effect.

First, we assume an orbital state where the energy gap of the $t_{2 g}$ orbital is smaller than the thermal excitation energy. In this case, the $t_{2 \mathrm{~g}}$ orbital can be regarded as triple degenerate, and $\varphi_{1}, \varphi_{2}$, and $\varphi_{3}$ orbitals are occupied by the two $3 \mathrm{~d}$ electrons with equal probability. The VED was simulated as $\frac{2}{3}\left|\varphi_{1}\right|^{2}+\frac{2}{3}\left|\varphi_{2}\right|^{2}+\frac{2}{3}\left|\varphi_{3}\right|^{2}$ (Fig. 2A). Next, we assumed the orbital state calculated using $Q$-mode analysis, in which the degenerate orbitals are stabilized. Because the two $3 \mathrm{~d}$ electrons occupy the degenerated $\varphi_{2}$ and $\varphi_{3}$, the VED is described as $\left|\varphi_{2}\right|^{2}+$ $\left|\varphi_{3}\right|^{2}$, as shown in Fig. 2B. The simulated VEDs do not reproduce the observed electron density (Fig. 2D). Next, we focused on the orbital state calculated by the point-charge model. In simple consideration, the two $3 \mathrm{~d}$ electrons form a spin singlet on stabilized $\varphi_{1}$, but it does not satisfy $S=1$ at the V site. In general, the energy gap of the $t_{2 g}$ orbitals due to the $D_{3 \mathrm{~d}}$ distortion is small, and Hund's rules are considered. In this case, one electron occupies the lower $\varphi_{1}$, and the other one is lifted to the degenerate orbitals and occupies either $\varphi_{2}$ or $\varphi_{3}$. This electron configuration satisfies $S=1$. In addition, the simulated VEDs such that the lifted electrons occupy only $\varphi_{2}$ or $\varphi_{3}$ orbital states also did not reproduce the observed VED (Fig. S2) [see ESI $\dagger$ Section 2 for details]. Therefore, we assumed that

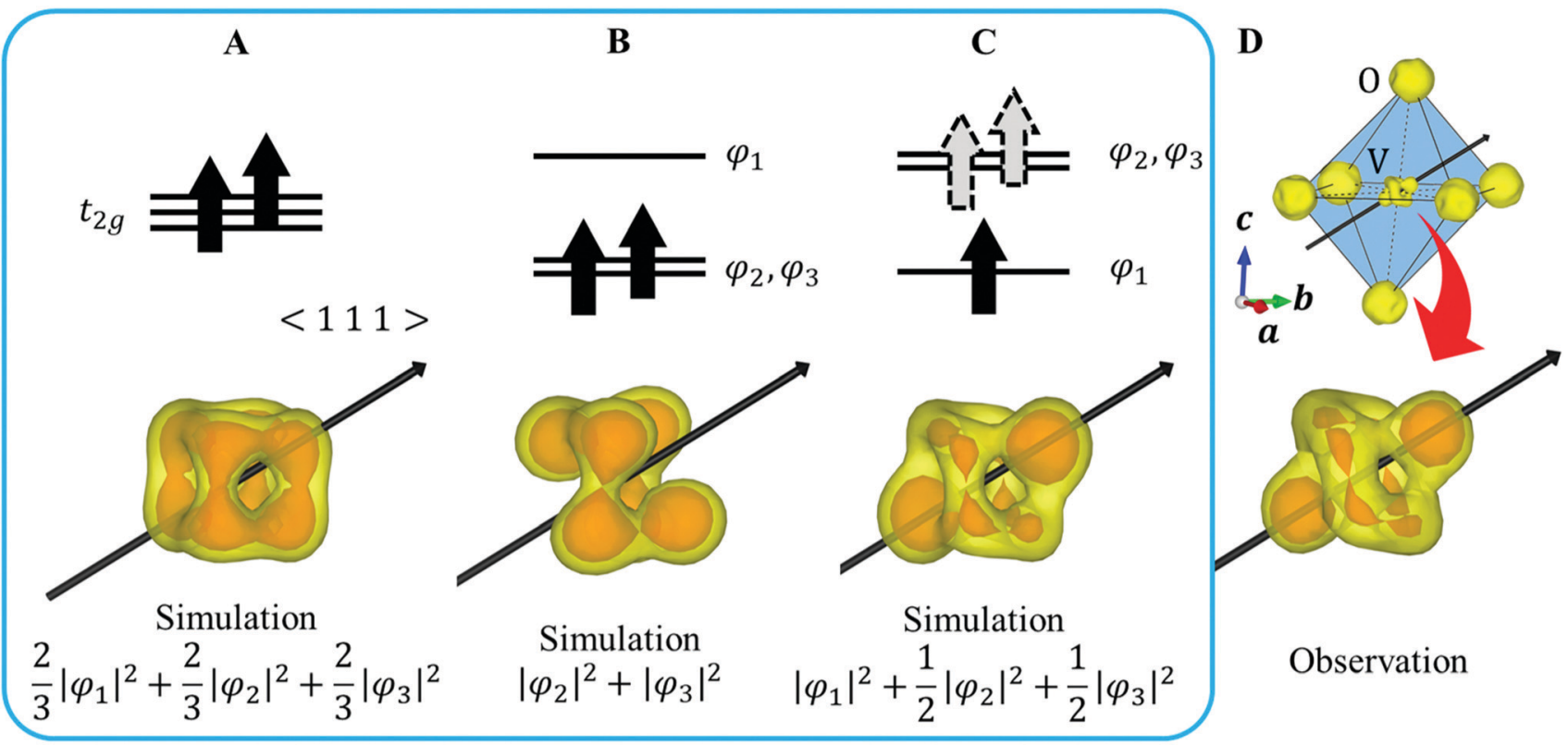

Fig. 2 (A-C) Assumed electron configurations and simulated VED of $V$ site. The red part of the VED highlights the characteristic components. The grey dotted arrows in (C) indicate that one electron on double degenerate orbitals occupies both orbitals with equal probability. (D) (Upper panel) Observed $V E D$ of $\mathrm{VO}_{6}$ octahedron with internal coordinates $(1 / 21 / 21 / 2)$ (Isosurface level $4.5 e / \AA^{3}$ ). The solid line indicates the octahedron, and the dotted line indicates the $\mathrm{V}-\mathrm{O}$ bonds. (Lower panel) Observed VED at $\mathrm{V}$ site. (Isosurface level $4.5 e / \AA^{3}$ (yellow), $5.3 e / \AA^{3}$ (red)). (A) Crystal structure of FeV $\mathrm{O}_{4}$ in the cubic phase. (B) The orbital states of $\mathrm{V}$ and $\mathrm{Fe}$ in the cubic phase. The black arrow in $\mathrm{VO}_{6}$ indicates the $\langle 111\rangle$ direction. 
the electrons on the double degenerate orbitals occupied both orbitals with equal probability. The simulation as $\left|\varphi_{1}\right|^{2}+\frac{1}{2}\left|\varphi_{2}\right|^{2}+\frac{1}{2}\left|\varphi_{3}\right|^{2}$ resulted in the VED distribution shown in Fig. 2C, which perfectly reproduced the experimental result. This result clearly means one electron occupies the lowest $\varphi_{1}$, and another electron is on the degenerate orbitals composed of $\varphi_{2}$ and $\varphi_{3}$. VED analysis by the CDFS method found that the degenerate orbitals reflect the anisotropy of their constituent orbitals in the cubic phase.

Here, we reconsider the degenerate orbital. As described above, the double degenerate orbital wavefunctions of $t_{2 g}$ are not uniquely described. The wavefunctions can also be represented as follows with an arbitrary phase value $\theta$ :

$$
\left\{\begin{array}{l}
\varphi_{2}^{\prime}(\boldsymbol{r})=\varphi_{2}(\boldsymbol{r}) \cos \theta+\varphi_{3}(\boldsymbol{r}) \sin \theta \\
\varphi_{3}^{\prime}(\boldsymbol{r})=-\varphi_{2}(\boldsymbol{r}) \sin \theta+\varphi_{3}(\boldsymbol{r}) \cos \theta
\end{array}\right.
$$

$\varphi_{1}, \varphi_{2}^{\prime}$, and $\varphi_{3}^{\prime}$ are orthogonal to each other. The orbital degrees of freedom mean that $\theta$ fluctuates in time and site $j$ as $\theta_{j}(t)$. Fig. 3 shows the schematic image of the fluctuating orbital. However, the VED obtained by XRD measurements was spaceand time-averaged. The image in which the electron on the degenerate orbital occupies both orbitals with equal probability is a simplified representation. The observed electron density $\rho_{\nu}$ with the fluctuated orbital states within the Born-Oppenheimer approximation is

$$
\rho_{v}=\frac{1}{N} \sum_{j}^{\text {crystal }}\left(\left|\varphi_{1, j}\right|^{2}+\left|\varphi_{2, j} \cos \theta_{j}(t)+\varphi_{3, j} \sin \theta_{j}(t)\right|^{2}\right)
$$

Here, $N$ is the number of $\mathrm{V}$ sites in the crystal, $j$ is the label of the $\mathrm{V}$ sites in the entire crystal, and $t$ is the time. The linear combination wavefunction of the degenerate orbitals is realized at each V site. However, the simulated VEDs at each V site do not suffice the site symmetry at the V site $(. \overline{3} m)$ (Fig. S3, ESI $\dagger$ ). The average of the sum over the entire crystal is observed as the

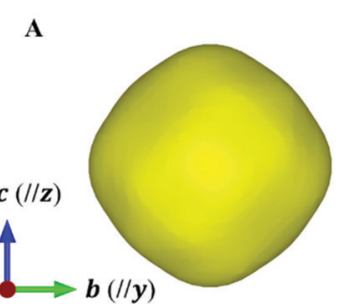

Observation

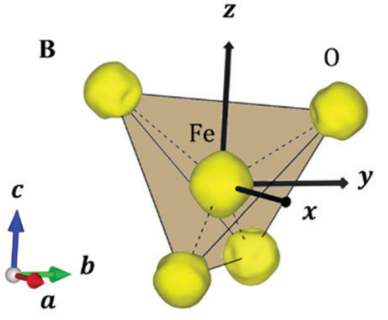

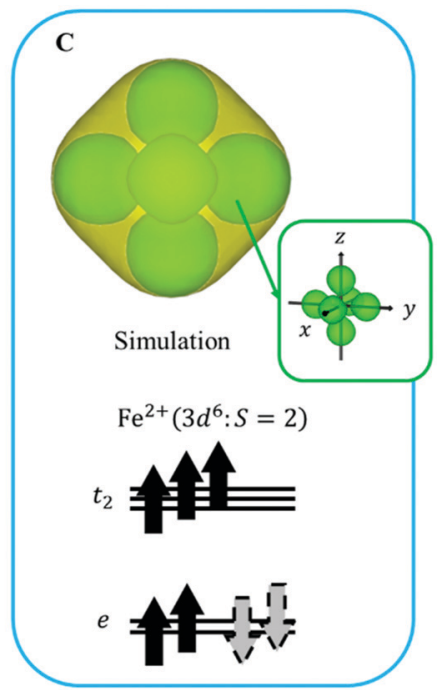

Fig. 4 (A) Observed VED at Fe site (Isosurface level 4.5e/ $\AA^{3}$ ). (B) Observed VED of $\mathrm{FeO}_{4}$ tetrahedron with internal coordinates (1/8 1/8 1/8) (Isosurface level $\left.4.5 e / \AA^{3}\right)$. The solid line indicates the tetrahedron, and the dotted line indicates the $\mathrm{Fe}-\mathrm{O}$ bonds. The black arrow indicates the quantum axes. (C) The simulated VED assumes the electron configuration of the bottom figure. The green part of the simulation corresponds to the VED of "downspin electron" on the degenerated orbitals. The anisotropic shape of the green part is shown in the inset. The grey dotted arrow in the electron configuration indicates that one electron on double degenerate e orbitals occupies both orbitals with equal probability.

electron density. The average is equivalent to:

$$
\begin{gathered}
\frac{1}{2 \pi} \int_{0}^{2 \pi}\left(\left|\varphi_{1}\right|^{2}+\left|\varphi_{2} \cos \theta+\varphi_{3} \sin \theta\right|^{2}\right) \mathrm{d} \theta \\
=\left|\varphi_{1}\right|^{2}+\frac{1}{2}\left|\varphi_{2}\right|^{2}+\frac{1}{2}\left|\varphi_{3}\right|^{2}
\end{gathered}
$$

Next, we focused on the Fe sites. When the VED of the CDFS analysis is viewed from the $\boldsymbol{a}$-axis, the VED has a slight anisotropy extending in the direction of lattice axes $\boldsymbol{a}, \boldsymbol{b}$, and $c$ at $160 \mathrm{~K}$ (Fig. 4A). Applying the same analogy as that applied to the $\mathrm{V}$ site, the $\mathrm{Fe}^{2+}-3 \mathrm{~d}^{6}$ orbital state should be understood from the VED. The anisotropy of the VED is responsible for
A
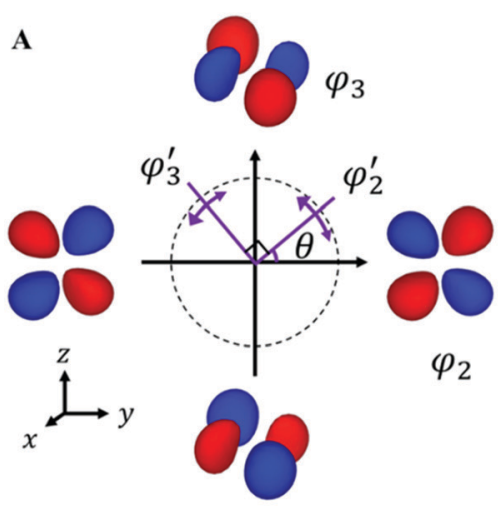

B

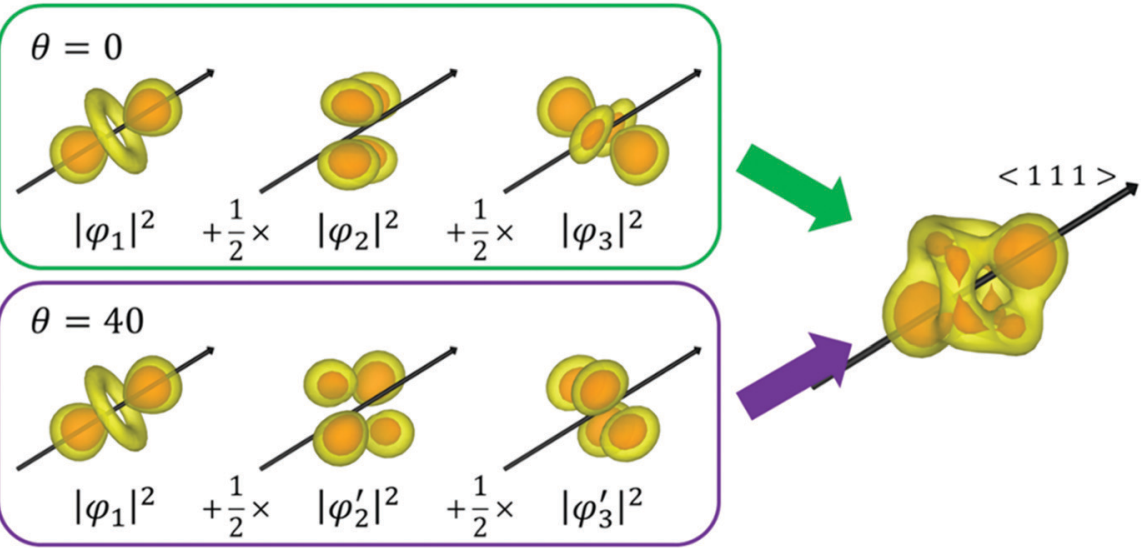

Fig. 3 (A) Schematic of the fluctuating orbital. The red and blue parts indicate the phase of the wavefunction. (B) Simulation with different $\theta$. Simulated VEDs have the same anisotropy even if $\theta$ is different. 
"down-spin electron" on the e orbitals. In cubic phase, $\mathrm{FeO}_{4}$ is a regular tetrahedron that satisfies the $\overline{4} 3 \mathrm{~m}$ site symmetry at the Fe site, and the e orbitals remain degenerate. The two wave functions of the e orbital of Fe can be expressed as:

$$
\left\{\begin{array}{l}
\psi_{1}(\boldsymbol{r})=\left|3 z^{2}-r^{2}\right\rangle . \\
\psi_{2}(\boldsymbol{r})=\left|x^{2}-y^{2}\right\rangle .
\end{array}\right.
$$

Similar to that for the V site, the wavefunction of "down-spin electron" can also be described as follows using an arbitrary phase value $\theta^{\prime}$.

$$
\psi^{\prime}(\boldsymbol{r})=\psi_{1}(\boldsymbol{r}) \cos \theta^{\prime}+\psi_{2}(\boldsymbol{r}) \sin \theta^{\prime}
$$

Because this electron exists on degenerate e orbitals, the averaged orbital state, which is $\frac{1}{2}\left|\psi_{1}\right|^{2}+\frac{1}{2}\left|\psi_{2}\right|^{2}$, is realized (Fig. 4C). Although the anisotropy of the VED around $\mathrm{Fe}$ is smaller than that around $\mathrm{V}$, the electron configuration of $\mathrm{Fe}^{2+}$ $3 \mathrm{~d}^{6}$ is well reproduced in real space.

Since cubic-tetragonal structural transition in $\mathrm{FeV}_{2} \mathrm{O}_{4}$ occurs at $140 \mathrm{~K}$, it is obvious that the electron orbitals have temporal fluctuations in cubic phase. Depending on the orbital fluctuations, a lattice fluctuation occurs due to the electron-lattice interaction. This phase transition is predicted to be mainly due to Fe orbital order. ${ }^{18}$ To confirm the orbital states below the phase transition temperature, direct observation is necessary. In the high-temperature tetragonal phase, a precise structural analysis of single crystals was performed, paying attention to the domain formation and site symmetry reduction [see ESI $\dagger$ Section 3]. The structural analysis at $120 \mathrm{~K}$ shows that the $\mathrm{VO}_{6}$ octahedron and $\mathrm{FeO}_{4}$ tetrahedron are slightly deformed, particularly $\mathrm{FeO}_{4}$ is compressed along the c-axis. This means a "down-spin electron" of Fe occupies the $\psi_{1}$ orbital due to Fe orbital order. In fact, when VED was extracted by using CDFS method, the anisotropy of $\mathrm{Fe}^{2+}-3 \mathrm{~d}^{6}$ including $\psi_{1}$ orbital asymmetry was observed (Fig. S4, ESI $\dagger$ ), but the anisotropy of the Vsite orbital was maintained (Fig. S5, ESI $\dagger$ ). When the orbital degeneracy of $\mathrm{Fe}$ is resolved, the symmetry of $\mathrm{FeO}_{4}$ is lowered due to $J T$ strain, but it is interesting to see how the phonons behave when the orbital degrees of freedom at the $\mathrm{V}$ site is preserved.

\section{Analysis result of inelastic X-ray scattering (IXS)}

The distortion in the form of compression/elongation of the $\mathrm{FeO}_{4}$ tetrahedron coupled with the wave function in eqn (7) corresponds to the $E$-symmetry. The $8 \mathrm{FeO}_{4}$ tetrahedra existing in the unit cell can be divided into two types based on the reversed quantization axis (Fig. S6, ESI $\dagger$ ). When these two types of $\mathrm{FeO}_{4}$ are distorted in the in-phase, this phonon mode is called the $E_{\mathrm{g}}$ mode, and when they are in the anti-phase, this phonon mode is called the $E_{\mathrm{u}}$ mode. The IXS experiments were
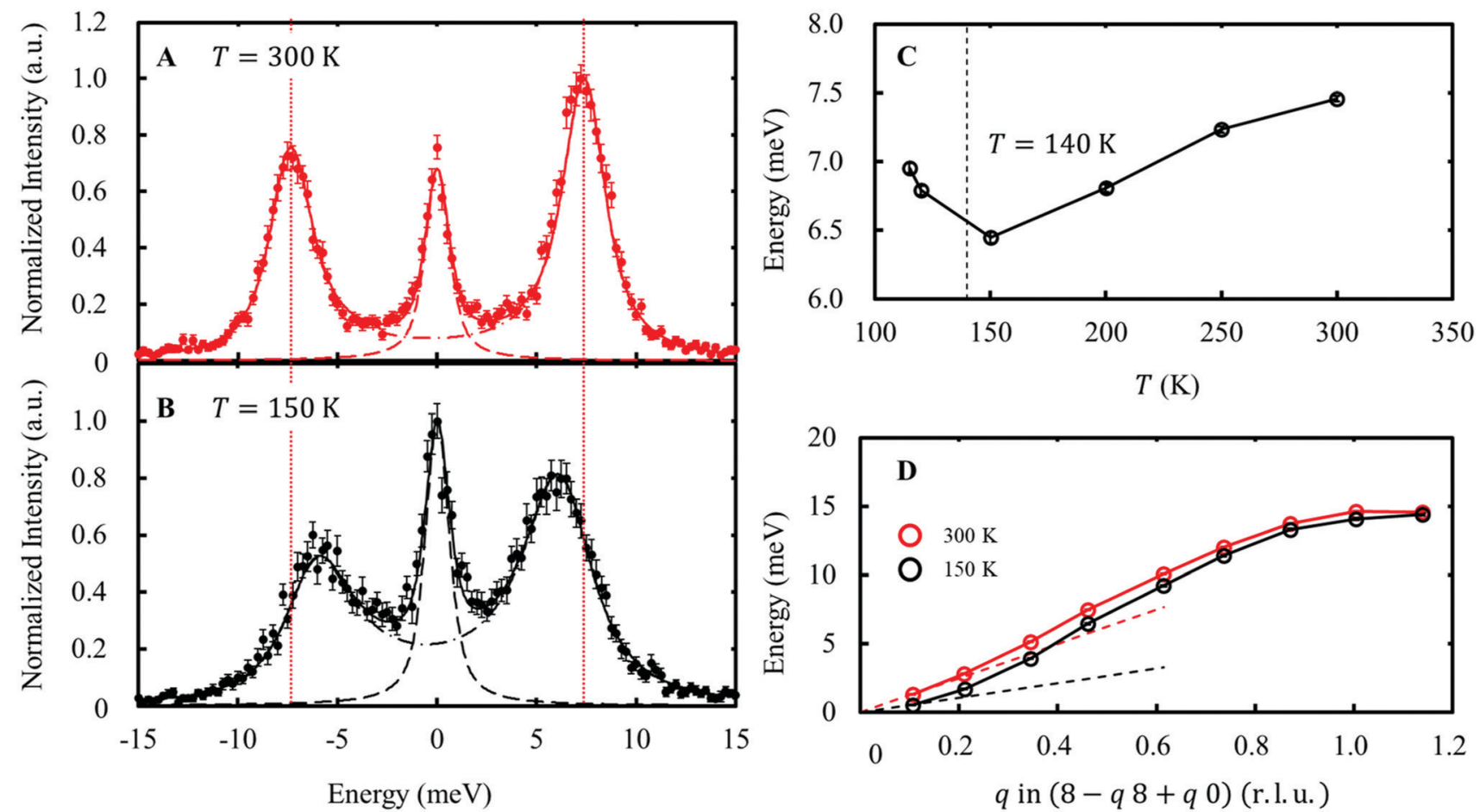

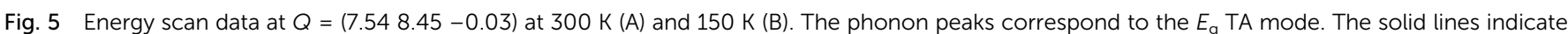

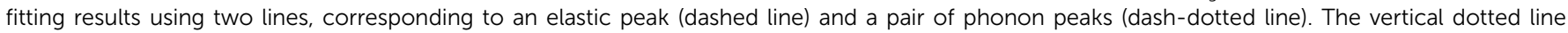

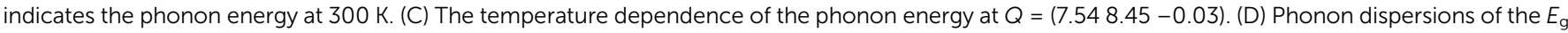

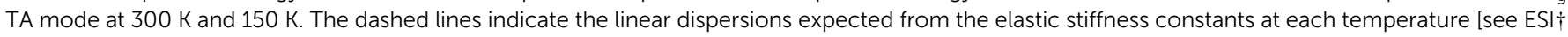
Section 2.3]. 
performed on the RIKEN BL43LXU beamline at SPring-8 [see ESI $\dagger$ Section 4, 5]. As a result, the slight softening of the transverse acoustic (TA) mode dispersing along the $\left[\begin{array}{lll}1 & 1 & 0\end{array}\right]$ and polarized along $\left[\begin{array}{lll}-1 & 1 & 0\end{array}\right]$, as shown in Fig. 5, indicates that the lattice distorts toward the transition point. This TA mode corresponds to the $E_{\mathrm{g}}$ mode. In contrast, the longitudinal optical (LO) mode dispersing and polarization along [ $\left.\begin{array}{lll}0 & 0 & 1\end{array}\right]$, corresponding to the $E_{\mathrm{g}}$ mode, was not observed due to weak intensity. Another LO mode corresponding to the $E_{\mathrm{u}}$ mode showed no temperature dependence in the range of $T=150$ 300 K (Fig. S9, ESI $\dagger$ ).

As shown in the dispersion diagram of the $E_{\mathrm{g}}$ TA mode in Fig. 5C, softening of the $E_{\mathrm{g}}$ TA mode is observed up to $q$ $\sim 0.7$ r.l.u. (approximately $8.5 \AA$ in real space). This softening is different from the usual precursor phenomenon of structural phase transitions. This is because the deformation mode, which should be coupled to orbital fluctuations, preserves the orbital degrees of freedom at the $\mathrm{V}$ site. This observed dispersion closely resembles the nematic state of iron-based superconductors, in which orbital degrees of freedom play an important role ${ }^{33}$ This result may be related to the distribution of the electron-lattice interaction when the orbital degrees of freedom are mixed at several different sites. To address this issue, we plan to conduct research on the same in the future. [see ESI† Section 6].

\section{Conclusions}

It was found that the VED with orbital degrees of freedom in $\mathrm{FeV}_{2} \mathrm{O}_{4}$ maintained a state reflecting orbital anisotropy even at relatively high temperatures. By directly observing the VED of the cubic phase, the orbital state can be directly determined without the need to estimate the crystal field or Hund's coupling constant due to the distortion of the $\mathrm{VO}_{6}$ octahedron. We showed that such orbital states are likely to be universal in the high-symmetry phase and that the quantum parameters can be determined directly by simply combining them with the CDFS method, provided that the accuracy of the diffraction measurements is ensured. Understanding the electronic states with orbital degrees of freedom will provide new prospects to reveal physical properties.

\section{Author contributions}

T. M., S. K., and H. S. designed and coordinated this study. S. N., and T. K. synthesized the samples. T. M., S. K., and H. S. performed the XRD and the IXS experiments. K. H., and D. I. supported the XRD and the IXS experiments the as the beamline scientist. T. H. performed the first-principles calculations of phonon. T. H., and A. B. discussed the IXS experimental results. N. K., Y. N., T. A., and J. N. advised this study. T. M., and H. S. wrote the manuscript. All authors discussed the experimental results and contributed to the manuscript.

\section{Conflicts of interest}

There are no conflicts to declare.

\section{Acknowledgements}

We thank K. Kojima, T. Hara, and Y. Hosogi for supporting the IXS experiments, Y. Wakabayashi, H. Fukui, and K. Siratori, for their fruitful discussions. The synchrotron radiation experiments were performed at SPring- 8 with the approval of the Japan Synchrotron Radiation Research Institute (JASRI) (Proposal No. 2019A0070-2021A0070/BL02B1, 2020A0835/BL43LXU, and 2021A1575/BL43LXU). The work leading to these results has received funding from Yamada Science Foundation.

\section{References}

1 Y. Tokura and N. Nagaosa, Orbital physics in transitionmetal oxides, Science, 2000, 288, 462-468.

2 K. I. Kugel' and D. I. Khomskī̄, The Jahn-Teller effect and magnetism: transition metal compounds, Sov. Phys. Usp., 1982, 25, 231-256.

3 A. J. Millis, Lattice effects in magnetoresistive manganese perovskites, Nature, 1998, 392, 147-150.

4 E. Dagotto, Complexity in strongly correlated electronic systems, Science, 2005, 309, 257-262.

5 A. Georges, L. d e'Medici and J. Mravlje, Strong Correlations from Hund's Coupling, Annu. Rev. Condens. Matter Phys., 2013, 4, 137-178.

6 J. Itatani, J. Levesque, D. Zeidler, H. Niikura, H. Pépin, J. C. Kieffer, P. B. Corkum and C. M. Villeneuve, Tomographic imaging of molecular orbitals, Nature, 2004, 432, 867-871.

7 P. Puschnig, S. Berkebile, A. J. Fleming, G. Koller, K. Emtsev, T. Seyller, J. D. Riley, C. Ambrosch-Draxl, F. P. Netzer and M. G. Ramsey, Reconstruction of molecular orbital densities from photoemission data, Science, 2009, 326, 702-706.

8 Y. Murakami, J. P. Hill, D. Gibbs, M. Blume, I. Koyama, M. Tanaka, H. Kawata, T. Arima, Y. Tokura, K. Hirota and Y. Endoh, Resonant X-Ray Scattering from Orbital Ordering in $\mathrm{LaMnO}_{3}$, Phys. Rev. Lett., 1998, 81, 582-585.

9 H. Yavas, M. Sundermann, K. Chen, A. Amorese, A. Severing, H. Gretarsson, M. W. Haverkort and L. H. Tjeng, Direct imaging of orbitals in quantum materials, Nat. Phys., 2019, 15, 599.

10 S. Kitou, T. Manjo, N. Katayama, T. Shishidou, T. Arima, Y. Taguchi, Y. Tokura, T. Nakamura, T. Yokoyama, K. Sugimoto and H. Sawa, Collapse of the simple localized $3 \mathrm{~d}^{1}$ orbital picture in Mott insulator, Phys. Rev. Res., 2020, 2, 033503.

11 S. Kitou, Y. Hosogi, R. Kitaura, T. Naito, T. Nakamura and H. Sawa, Direct Observation of Molecular Orbitals Using Synchrotron X-ray Diffraction, Crystals, 2020, 10, 998.

12 H. Sawa, Y. Wakabayashi, Y. Murata, M. Murata and K. Komatsu, Floating Single Hydrogen Molecule in an Open-Cage Fullerene, Angew. Chem., 2005, 117, 2017-2019. 
13 S. Aoyagi, E. Nishibori, H. Sawa, K. Sugimoto, M. Tanaka, Y. Miyata, R. Kitaura, H. Shinohara, H. Okada, T. Sakai, Y. Ono, K. Kawachi, S. Yokoo, S. Ono, K. Omote, Y. Kasama, S. Ishikawa, T. Komuro and H. Tobita, layered ionic crystal of polar Li@C $\mathrm{C}_{60}$ superatoms, Nat. Chem., 2010, 2, 678.

14 E. J. W. Verway, Electronic Conduction of Magnetite $\left(\mathrm{Fe}_{3} \mathrm{O}_{4}\right)$ and its Transition Point at Low Temperature, Nature, 1939, 144, 327-328.

15 S. H. Lee, H. Takagi, D. Louca, M. Matsuda, S. Ji, H. Ueda, Y. Ueda, T. Katsufuji, J. H. Chung, S. Park, S. W. Cheong and C. Broholm, Frustrated Magnetism and Cooperative Phase Transition in Spinels, J. Phys. Soc. Jpn., 2010, 79, 011004.

16 T. Katsufuji, T. Suzuki, H. Takei, M. Shingu, K. Kato, K. Osaka, M. Tanaka, H. Sagayama and T. Arima, Structural and Magnetic Properties of Spinel $\mathrm{FeV}_{2} \mathrm{O}_{4}$ with Two Ions Having Orbital Degrees of Freedom, J. Phys. Soc. Jpn., 2008, 77, 053708.

17 G. J. MacDougall, V. O. Garlea, A. A. Aczel, H. D. Zhou and E. Nagler, Magnetic order and ice rules in the multiferroic spinel $\mathrm{FeV}_{2} \mathrm{O}_{4}$, Phys. Rev. B: Condens. Matter Mater. Phys., 2012, 86, 060414(R).

18 Y. Nii, H. Sagayama, A. Aoyagi, R. Sakai, S. Maki, E. Nishibori, H. Sawa, K. Sugimoto, H. Ohsumi and M. Takata, Orbital structures in spinel vanadates $\mathrm{AV}_{2} \mathrm{O}_{4}(\mathrm{~A}=\mathrm{Fe}, \mathrm{Mn})$, Phys. Rev. B: Condens. Matter Mater. Phys., 2012, 86, 125142.

19 A. Kismarahardja, J. S. Brooks, H. D. Zhou, E. S. Choi, K. Matsubayashi and Y. Uwatoko, Dielectric properties of single crystal spinels in the series $\mathrm{FeV}_{2} \mathrm{O}_{4}, \mathrm{MnV}_{2} \mathrm{O}_{4}$, and $\mathrm{CoV}_{2} \mathrm{O}_{4}$ in high magnetic fields, Phys. Rev. B: Condens. Matter Mater. Phys., 2013, 87, 054432.

20 S. Nakamura and A. Fuwa, Distinct Evidence of Orbital Order in Spinel Oxide $\mathrm{FeV}_{2} \mathrm{O}_{4}$ by ${ }^{57} \mathrm{Fe}$ Mössbauer Spectroscopy, J. Phys. Soc. Jpn., 2016, 85, 014702.

21 B. R. Myoung, S. J. Kim, J. T. Lim, T. Kouh and C. S. Kim, Microscopic evidence of magnetic and structure phase transition in multiferroic spinel $\mathrm{FeV}_{2} \mathrm{O}_{4}, A I P A d v$., 2017, 7, 055828.

22 Q. Zhang, M. Ramazanoglu, S. Chi, Y. Liu, T. A. Lograsso and D. Vaknin, Magnetic excitations and anomalous spinwave broadening in mutiferroic $\mathrm{FeV}_{2} \mathrm{O}_{4}$, Phys. Rev. B: Condens. Matter Mater. Phys., 2014, 89, 224416.
23 K. Matsuura, H. Sagayama, Y. Nii, N. D. Khanh, N. Abe and T. Arima, X-ray magnetic circular dichroism of an orbital ordered state in the spinel-type vanadium oxide $\mathrm{AV}_{2} \mathrm{O}_{4}$ (A = Mn, Fe), Phys. Rev. B: Condens. Matter Mater. Phys., 2015, 92, 035133.

24 D. Dey, T. Maitra and A. Taraphder, Orbital ordering in $\mathrm{Fe}_{1-\mathrm{x}} \mathrm{Mn}_{\mathrm{x}} \mathrm{V}_{2} \mathrm{O}_{4}$ : A first-principles study, Phys. Rev. B, 2016, 93, 195133.

25 H. Mamiya, M. Onoda, T. Furubayashi, J. Tang and I. Nakatani, Structural and magnetic studies on vanadium spinel $\mathrm{MgV}_{2} \mathrm{O}_{4}$, J. Appl. Phys., 1997, 81, 5289-5291.

26 Y. Ueda, N. Fujiwara and H. Yasuoka, Magnetic and Structural Transitions in $\left(\mathrm{Li}_{\mathrm{x}} \mathrm{Zn}_{1-\mathrm{x}}\right) \mathrm{V}_{2} \mathrm{O}_{4}$ with the Spinel Structure, J. Phys. Soc. Jpn., 1997, 66, 778-783.

27 M. Onoda and J. Hasegawa, A distortion of pseudotetramers coupled with the Jahn-Teller effect in the geometrically frustrated spinel system $\mathrm{CdV}_{2} \mathrm{O}_{4}, J$. Phys.: Condens. Matter, 2003, 15, L95-L102.

28 R. Plumier and M. Sougi, Observation of a first order transition in the ferrimagnetic spinel $\mathrm{MnV}_{2} \mathrm{O}_{4}$, Solid State Commun., 1987, 64, 53-55.

29 A. Kismarahardja, J. S. Brooks, A. Kiswandhi, K. Matsubayashi, R. Yamanaka, Y. Uwatoko, J. Whalen, T. Siegrist and H. D. Zhou, $\mathrm{Co}\left[\mathrm{V}_{2}\right] \mathrm{O}_{4}$ : A Spinel Approaching the Itinerant Electron Limit, Phys. Rev. Lett., 2011, 106, 056602.

30 S. Ohtani, Y. Watanabe, M. Saito, N. Abe, K. Taniguchi, H. Sagayama, T. Arima, M. Watanabe and Y. Noda, Orbital dilution effect in ferrimagnetic $\mathrm{Fe}_{1-\mathrm{x}} \mathrm{Mn}_{\mathrm{x}} \mathrm{Cr}_{2} \mathrm{O}_{4}$ : competition between anharmonic lattice potential and spin-orbit coupling, J. Phys.: Condens. Matter, 2010, 22, 176003.

31 K. Siratori and K. Kohn, Ground State of a de Electron in an Elastic Octahedron of Ligands, J. Phys. Soc. Jpn., 2010, 79, 114720.

32 P. P. Ewald, Die Berechnung optischer und elektrostatischer Gitterpotentiale, Ann. Phys., 1921, 369, 253-287.

33 A. M. Merritt, F. Weber and J. P. Castellan, Th. Wolf, D. Ishikawa, A. H. Said, A. Alatas, R. M. Fernandes, A. R. R. Baron, D. Reznik, Nematic Correlation Length in IronBased Superconductors Probed by Inelastic X-Ray Scattering, Phys. Rev. Lett., 2020, 124, 157001. 